
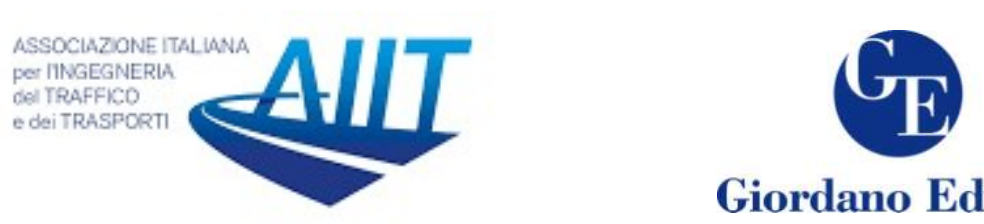

Giordano Editore

\title{
BIM for infrastructure: an efficient process to achieve 4D and $5 D$ digital dimensions
}

\author{
Fabrizio D'Amico', Luca D'Ascanio', Maria Chiara De Falco', \\ Chiara Ferrante ${ }^{1}$, Davide Presta ${ }^{1}$, Fabio Tosti ${ }^{2}$ \\ ${ }^{1}$ Department of Engineering, Roma Tre University, Via Vito Volterra 62, 00146, Rome, Italy \\ ${ }^{2}$ School of Computing and Engineering, University of West London (UWL), St Mary's Road, Ealing, W5 \\ $5 R F$, London, $U K$
}

\begin{abstract}
Introduction of the Building Information Modeling (BIM) approach in Civil Engineering practices allows a design optimization through both an improved control of all the components of a project and an increased efficiency in the collaboration between all professionals involved. In Italy, major attention is being paid by scientists and professionals on this subject area, especially after the implementation of the national law DM $560 / 2017$. This document will gradually rule the integration of BIM-based procedures for contractors in major public tenders. The present paper aims to propose a methodology for implementing an optimization dynamic model of time (4D) and costs (5D) across different project phases. To achieve this aim, the process relies on several authoring tools and 4D/5D modeling software, capable to incorporate a variety of project data in a unique and shared environment. The most effective methodology to maximize the interoperability between various codes will be presented. In this regard, a dynamic model involving an optimizationoriented approach for both the quantity surveying and the definition of the design management timeline is introduced. It is important to emphasize that these aspects are two of the major issues for the evaluation criteria in a civil engineering project. Also, this bidirectional process allows for a more accurate estimation of project costs as well as to achieve an optimized timeline of construction activities. Finally, it worth reminding that in case of constrained changes during the design phase, a dynamic model permits to effectively reiterate the process with a significant reduction of the overall computational time.
\end{abstract}

Keywords: Project Management; Infrastructures; Building Information Modeling (BIM); Time and Cost Optimization.

\section{Introduction}

The Building Information Modelling (BIM) process has been progressively implemented worldwide in the Architecture, Engineering and Construction (AEC) areas in recent times (Eastman et al., 2001).

Development of BIM in civil engineering applications is fostered by a particular European political situation aiming to improve the efficiency standards of the construction industry. To that extent, the European Union Public Procurement Directive (EUPPD) (EU, 2014) has mentioned that "the 28 European Member States may

\footnotetext{
* Corresponding author: Fabrizio D’Amico (fabrizio.damico@uniroma3.it)
} 
encourage, specify or mandate the use of BIM for publicly funded construction and building projects in the European Union by 2016" (Wang et al., 2014; Sacks and Gurevich, 2016).

In Italy, BIM-based procedures for contractors have been integrated in major public tenders following the decree of the Ministry of Infrastructures and Transports (MIT, 2017). Target of this decree is to achieve a greater efficiency and effectiveness within the entire public work sector. The decree defines timetable and details for the progressive introduction of requirements within specific electronic methods and tools and identifies their application area. Furthermore, it imposes these methods and tools to be applied across all the aspects of construction design and the operation of civil engineering structures and infrastructures (e.g. bridges, buildings, etc).

It is well-known that BIM is a conceptual model that allows a parametric approach for storing all the available information related to a specific project within the proposed model. In fact, a wide range of information converge and collaborate to a civil structure design across the various design process phases. The level of detail (Levels Of Definition - LOD) of a BIM model increases with the project follow-up: in a first stage, the information contained in the model are usually limited to the preliminary project; on the contrary, the model passes in the following stages from a simple conceptual model to a virtual model (i.e., the "As-built" model) until it reaches the constructive level. Information on the LOD are required for all the objects in a BIM model within the different design phases.

A BIM Execution Plan describes the LOD that every BIM authoring discipline must guarantee by the set deadlines for each specific project. In more detail, seven levels of LOD are defined as a function of the geometry, graphic representation and attributes of an element.

To analyse in depth the multi-aspects of a design information for the control of the entire design process and to fulfil requirements of a BIM process, many other dimensions need to be added. According to Lee et al. (2005), the $n \mathrm{D}$ model is an extension of the 3D BIM model including multi-aspects of the design information required at each stage of the lifecycle of a building facility.

To achieve a better control of the project, the time factor quickly became the fourth dimension of the BIM environment (Lopez et al., 2016; Zhang and Hu, 2011). By using 4D BIM, it was possible to compare different plans by visualization of the work sequences and duration of each task. In addition, 4D BIM simulations can turn into 5D BIM simulations by incorporating information about the resource consumption. This can provide a better understanding of the utilization of resources over time. Nevertheless, it worth mentioning that only limited research is available nowadays about use of 5D BIM (Lu et al., 2016; Mitchell, 2012, Matejka and Vitasek, 2018).

As the fourth and fifth dimensions seem to have gained a certain rate of prominence, they will be explored in this study to optimize the entire design process. Therefore, a novel dynamic approach is proposed that will integrate time and cost information in a synergic system for supporting the design process.

The study is outlined as follows. Section 2 presents a background on the potentials of using 4D and 5D dimensions. The entire modelling process with the use of various authoring and simulation 4D and 5D software is discussed in Section 3. These are oriented towards the optimization of the quantity surveying as well as to the definition of the works timeline. Finally, conclusions and future perspectives about this novel approach are discussed in Section 4. 


\section{Background}

Time and cost variables are two of the most important parameters in a design process, especially for infrastructure design, and their definition is often a matter of political discussion. Considering time and cost variables in a project implies to solve a typical engineering problem by attempting to find the best solution in terms of efficiency and effectiveness. The optimum is achieved when the project output can maximize the quality and the effectiveness of a solution and minimize time and costs of execution.

From a practical point of view, an iterative process is required to optimize the design process of an infrastructure, starting from a proper design process, up to the provision of a proper quantity surveying, to end up with the definition of an effective timeline.

In regard to the development of the above-discussed subsequent phases within a traditional design approach, the quantity surveying and the timeline are closely related to the first design hypothesis on a one-way based dialogue. Therefore, any change in timeline does not correspond to any updates provided to the design parameters and the quantity surveying.

The novel approach presented in this study attempts to provide a solution to this problem. To this purpose, a dynamic model is proposed that can integrate time and cost variables in a bi-directional model able to simultaneously update i) the parameters of the proper design model, ii) the quantity surveying and iii) the timeline at any given modifications. The proposed model is based on currently available commercial tools which are employed to orient the design process towards the optimization of the project.

\section{Modeling}

Taking advantage of all the available features of BIM procedures and technologies, a model is built for managing and storing all the data of a transport infrastructure project. More specifically, the choice of a BIM-oriented software related to the proper design of the infrastructure, the quantity surveying and the timeline are handled.

Furthermore, a standard project work breakdown structure (WBS) is built and the procedure of time and cost implementation in the model is shown. Lastly, advantages of considering a dynamic model that involves information on time and costs of realization are discussed.

\subsection{Software}

\subsubsection{Infrastructure model}

At first, the process provides the choice of the authoring BIM-oriented software more suitable for the modelling of a transport infrastructure. Currently, the most used software are: Civil 3D (developed by Autodesk), OpenRoads (Bentley), Roads (Sierrasoft), all of which can manage effectively the infrastructure design and provide a valuable support to project designers. However, following an analysis of the tools offered by every BIMoriented software, Civil 3D has proven to be the most effective for this purpose. This software facilitates the integration between the structural/architectural elements, installations and the infrastructure axis, by allowing for the interchange of file formats. Furthermore, Civil 3D offers very useful tools for customization of the parameters to assign to every digital object of the model.

According to the above, the BIM model was prepared for the development of the subsequent phases of the process by means of the Civil 3D software. 


\subsubsection{Timeline}

The second phase relates to the choice of the software most fitting the scheduling of the work phases. Similarly to the infrastructure model, an analysis of all the tools available to achieve the relevant objective is carried out. The timeline software is required to manage the scheduling of the work and to be interoperable with the BIM model and the software that will manage the quantity surveying. For this purpose, the following software were considered: MS Project (developed by Microsoft), Primavera P6 (Oracle) and STR Vision CPM (Team System). It is important to note that not all software allows to import an Industry Foundation Classes (IFC) file (ISO, 2016). To tackle this problem, the possibility to connect the software was explored through the creation of interface masks. As a result, STR Vision CPM was selected as the timeline software in this study, due to the capability of importing IFC files and the easy in managing the timeline schedule process.

\subsubsection{Quantity surveying}

It is now necessary to define the software to draw up the bill of quantities. The most widespread software for quantity surveying are: Primus CAD and Primus IFC (both developed by ACCA software), STR Vision CPM (Team System).

After several tests performed with the three available software, Primus CAD did not seem to be the most appropriate tool for implementation in the infrastructure model in regard to the quantity surveying aspect for this particular project. Indeed, the visualised model does not account for the geometric properties of the road solid, as prepared in the informative model. Also, it does not allow to consider IFC standards and does not permit to compute the elements of the model and to associate them to any computation item.

On the other hand, despite Primus IFC considers both the IFC standards and the model properties, a major drawback concerns the $3 \mathrm{D}$ model visualization. To this effect, the reliability of the quantities interpreted by the model cannot be easily verified. The above is not valid for IFC models created with vertical authoring software. Nevertheless, this visualization limit was decisive in the choice of the model used for the application case presented in this paper.

On the contrary, STR Vision CPM allows to view and read data and quantities of the IFC model exported by the BIM-oriented authoring software. Due to this latter, it is possible to obtain an exact surveying that refers directly to the quantities of the information model of the infrastructure. The concerning procedures will be detailed later in the paper.

Once all the BIM-oriented software of the process have been identified, the steps to build a unique dynamic model, from which all the information about time and costs might be obtained, will have to be defined. In regard to the actual modelling phase, the technical aspects of the single software will be omitted, although a few considerations on the model preconditions in the subsequent phases are provided.

\subsection{Standard project WBS}

To ensure that the BIM model is effective, a unique identification code for each item must be defined. Furthermore, it could be useful to provide the code with a recognizable structure in order to compute the various elements separately. Therefore, the use of the standard project WBS seems to be the most suitable solution for this purpose. 


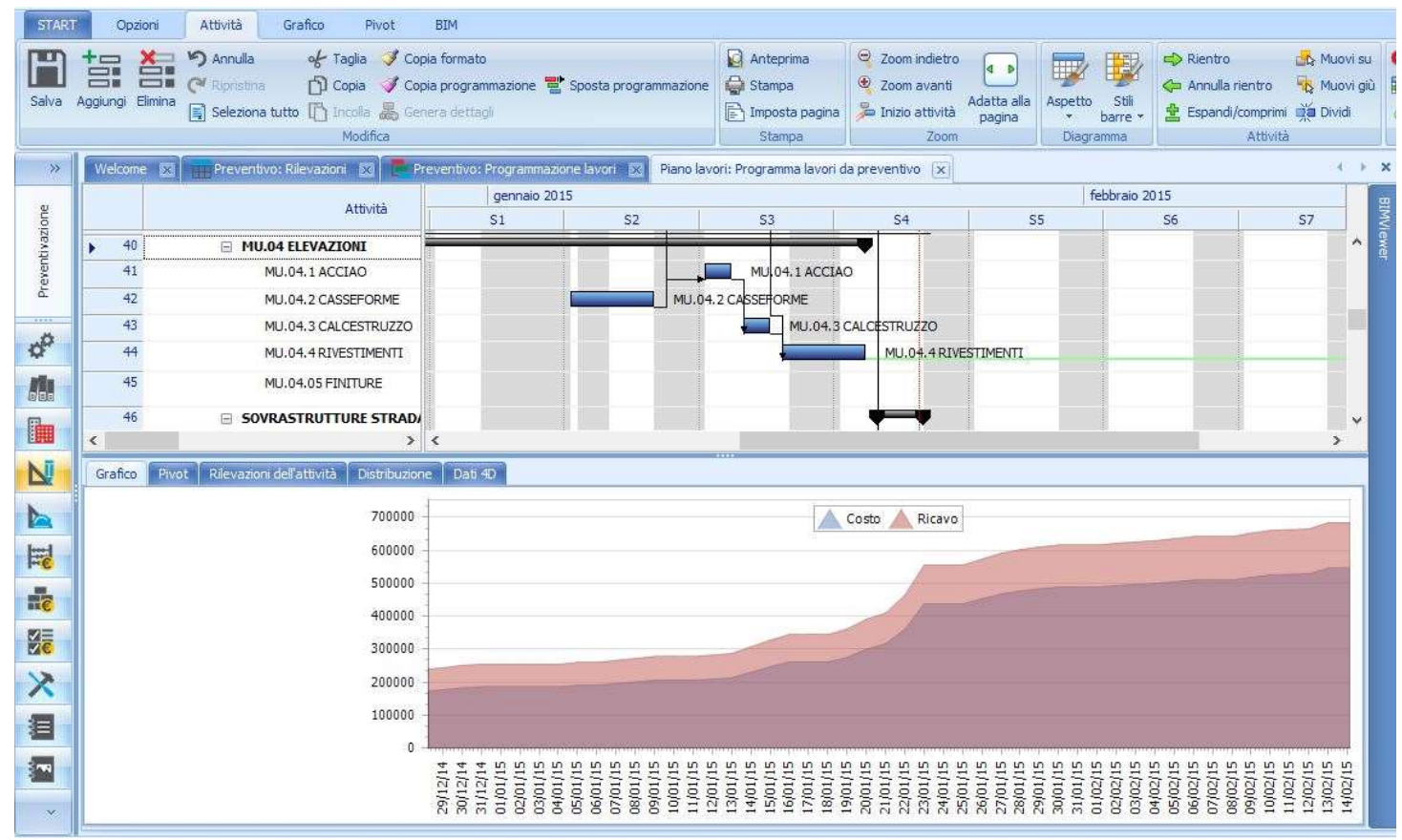

Figure 1: the 4D software interface.

Requirements of the project WBS are: i) uniqueness of the code for each element; ii) structure is based on several levels such as the macro-activities; iii) structure is able to filter elements realised at the same phase of the time schedule. Once the WBS code creation rules have been set, the BIM Coordinator will identify the shared parameters that every element of the model must possess (i.e., a parameter for each level of the WBS structure) and the BIM Specialist will provide values to each field of the different parameters with the code.

\subsection{Procedure}

\subsection{1 $4 \mathrm{D}$ - the time variable implementation}

The time variable implementation allows to link the different elements of the infrastructure model to the realisation phases identified in the timeline. This phase is made up of two sub-phases: the first one to be carried out in a project management software as STR Vision CPM (Fig. 1); the second one to be performed with a 4D simulation software such as Synchro (developed by Bentley), Navisworks (Autodesk) or Fuzor (Kalloc Studios).

The former sub-phase allows to create and analyse the timeline starting from the previously-defined WBS. This ensures full coherence between the items of the calculation and those in the project Gantt.

The second sub-phase provides to export the timeline from STR Vision CPM and import it in the simulation 4D software platform. In this regard, "dynamic interferences" are also analysed. It worth mentioning that these interferences are not related to the construction elements but they are due to the simultaneous presence of construction site vehicles in the same area for different activities. 
Since this analysis is not always immediate, it is necessary to refer again to the project WBS, which allows to use the same parameters employed for the calculation phase and to ensure consistency with rest of the documents.

\subsubsection{D - the cost variable implementation}

Implementation of the cost variable in the design BIM model follows the implementation of the time variable.

Up to this phase, the model contains a series of information on road element geometries, materials and the timeline for each activity that, by means of the WBS code, are associated with an item in the price list loaded in the computational software.

The IFC file is imported into the STR Vision CPM software. Quantities will be read from the loaded model and costs will be associated. A manual procedure and an automatic procedure can be carried out to associate the cost of any item from the list of costs.

The manual procedure requires the selection of model elements directly from the software internal viewer in order to quantify costs of each element as the product between the quantity read from the model and the item cost. By repeating this procedure for all the characteristic elements involved in the project, an overall calculation of the work costs is obtained. However, this procedure takes longer compared to the automatic one and exposes the user to a higher rate of errors in the selection stage of the elements.

The automatic procedure allows a dynamic update of the calculations and a minimization of the manual work with a reduction of the probability of error at each operation stage. This procedure is referred to the WBS code and it is based on filters.

The filtering rules of the elements known as "BIM calculation rules" must be set based on different levels of WBS depending on the type of activity. These rules allow an advanced search of the objects within the model through the property section containing the quantitative and qualitative information, the materials composing the structure and other further details. Using this function, it is possible to update the calculations instantly at every change of the project informative model. In addition to this, rules already defined can be used for other estimates. In fact, the program automatically recognizes the elements modified, deleted or added and dynamically updates measures, quantities and costs by reloading the IFC file, and through the use of the calculation updating function (Fig. 2).

It is important to underline that before importing the IFC file into the computational software, the validity of the file in all its parts must be assured by a model checking and integrity validation procedures, being it model-dependent.

\subsection{Dynamic model approach}

The procedure described above allows to have a dynamically-updated model according to basic composition factors, i.e., i) the basic informative model of the infrastructural work; ii) the time schedule; iii) the price list and iv) the project WBS. Within this context, it is important to note that the direct relationship between the bill of quantities and the time schedule is still lacking.

This will help to automate the process and, therefore, to build a dynamic model. Also, this is crucial at a more advanced stage of the design and construction process (for example the construction project), where the methodology used to perform a given processing task is fundamental for the definition of the calculations. 


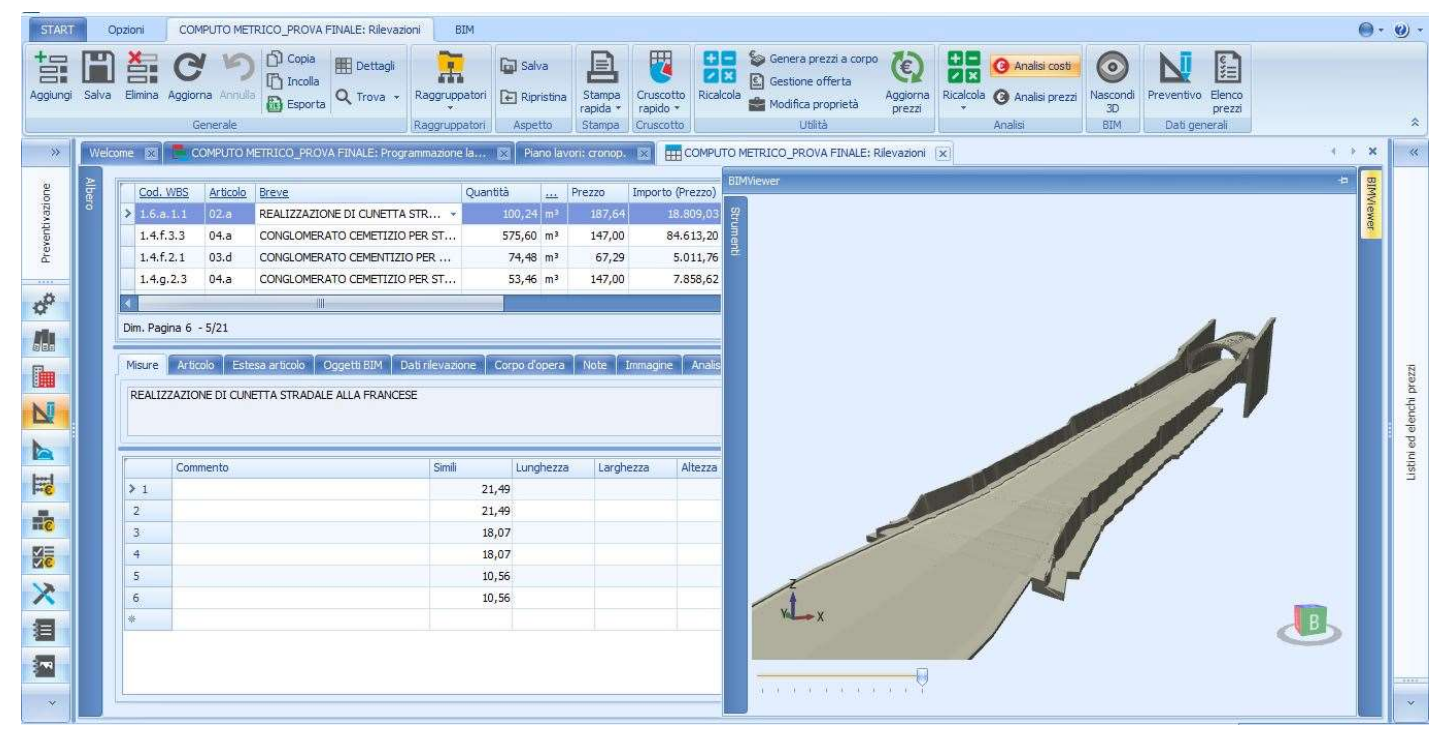

Figure 2: the 5D software interface.

To this effect, a methodology is proposed in this study to create a dependency between the bill of quantities (BoQ) and the time schedule. In order to create this process, it is necessary to further discretize the BoQ entries. It is here proposed to divide the BoQ into two sub-categories: BoQS (Bill of Quantities of Supplies) and BoQR (Bill of Quantities of Resources).

This is necessary to make the BoQ dependent on the model. Following this structure, the BoQS only depends on the quantities outcoming from the BIM model (technical project) whereas the BoQR solely depends on the time schedule, as it is built by multiplying the different resources identified by the time schedule for the daily cost.

The method assumes the creation of an informative model of the infrastructure. This complies with the project standards necessary for the preparation of the various elements for a subsequent valorisation phase with the multiple information previously discussed. The WBS code is one of the major information that must necessarily be entered. This can be obviously done only after defining a project WBS that meets the criteria already described in paragraph 3.2, which now becomes compulsory given the next use of this code in the automated procedure.

After creating the informative model and assigning a unique and standardized code to each element, it is possible to proceed with the construction of the BoQS. The first step is to import the BIM model created so far into the quantity surveying software.

Within the quantity surveying software, the fundamental step is the creation of an interface mask between the price list and the WBS codes. Using this association, it will be possible to link each item of the price list to each element of the model which, as already mentioned, will be characterized by a unique WBS code.

The assignment of the cost items at this stage will be automatic and precisely for this reason free of errors. It also worth reminding that the model with its elements and respective WBS codes must be verified very carefully, since an error in their modelling or compilation may result in an error on the BoQ.

At this stage, we are in possession of a model that allows to extract the information for drawing up a BoQS. To this purpose, it is possible to structure the document in different forms, according to the item costs to be grouped. Also, we can refer in this case to the WBS codes by combining items according to different levels of the WBS itself. 
The extraction of the BoQS at this stage is immediate, providing that a reference template has been prepared.

However, the BoQ is not yet complete as it has been divided into two parts, one related to supplies and one related to resources. The BoQR needs the definition of the time schedule to be constructed in the following way.

We will have to determine a rough work program that can be set starting from a subdivision of the WBS used so far. It is also necessary to define the quantity, productivity and daily costs of the resources available. This information can be modified and updated even further.

At this stage, it will be possible to assign the newly-defined resources to the different activities in the project management software and have the execution times of the various activities automatically calculated. This is possible by using the resource productivity information and the quantities readable from the model elements which are previously assigned to those resources.

As an example, the activity of building the foundation of a retaining wall, to which a working team composed of different vehicles and workers of known productivity and hourly cost are assigned as a resource, is considered. Using the project management software, the above-discussed procedure allows to read the quantity of concrete for realizing the foundations of the wall from the model.

This information, together with the team's productivity assigned to the activity, allows to calculate the time of execution.

This execution time will be then multiplied by the daily cost of the teamwork in order to define the labour cost for that specific activity.

To extend this rationale to all the activities, it is necessary to access the resource diagrams. As usual, it is possible to extract the Gantt and the resource diagrams. Using information derived from the latter, the software can quantify the working days of each resource by multiplying their number with the daily cost.

A computational structure will be created similarly to the case of the BoQS. Finally, adding the BoQR to the BoQS will lead to the complete Bill of Quantities.

The execution of the procedure described above allows a complete dynamism with respect to modifications concerning:

- $\quad$ The BIM model: at each change, the BoQS will be automatically updated, as well as the duration of the time schedule and consequently the BoQR. This will reflect into the BoQ;

- The time schedule: by modification of the logical order of activities in the schedule, the BoQR and therefore the BoQ will be updated automatically.

It should be noted that at each modification of the model, and therefore of the technical project, the time schedule will be updated only in terms of duration of the activities deriving from a different quantity of a specific element. Nevertheless, the logical order of the activities will not change as this process will always remain at discretion of the designer.

Similarly, it must be considered that it will not be possible to directly modify the duration of the activities in the timetable, since this procedure is completely automatic. This is due to the strict dependence between activity duration and the relative amount of resources assigned to them as well as their productivity.

A flow-chart of the procedure discussed above in given in Fig. 3. 


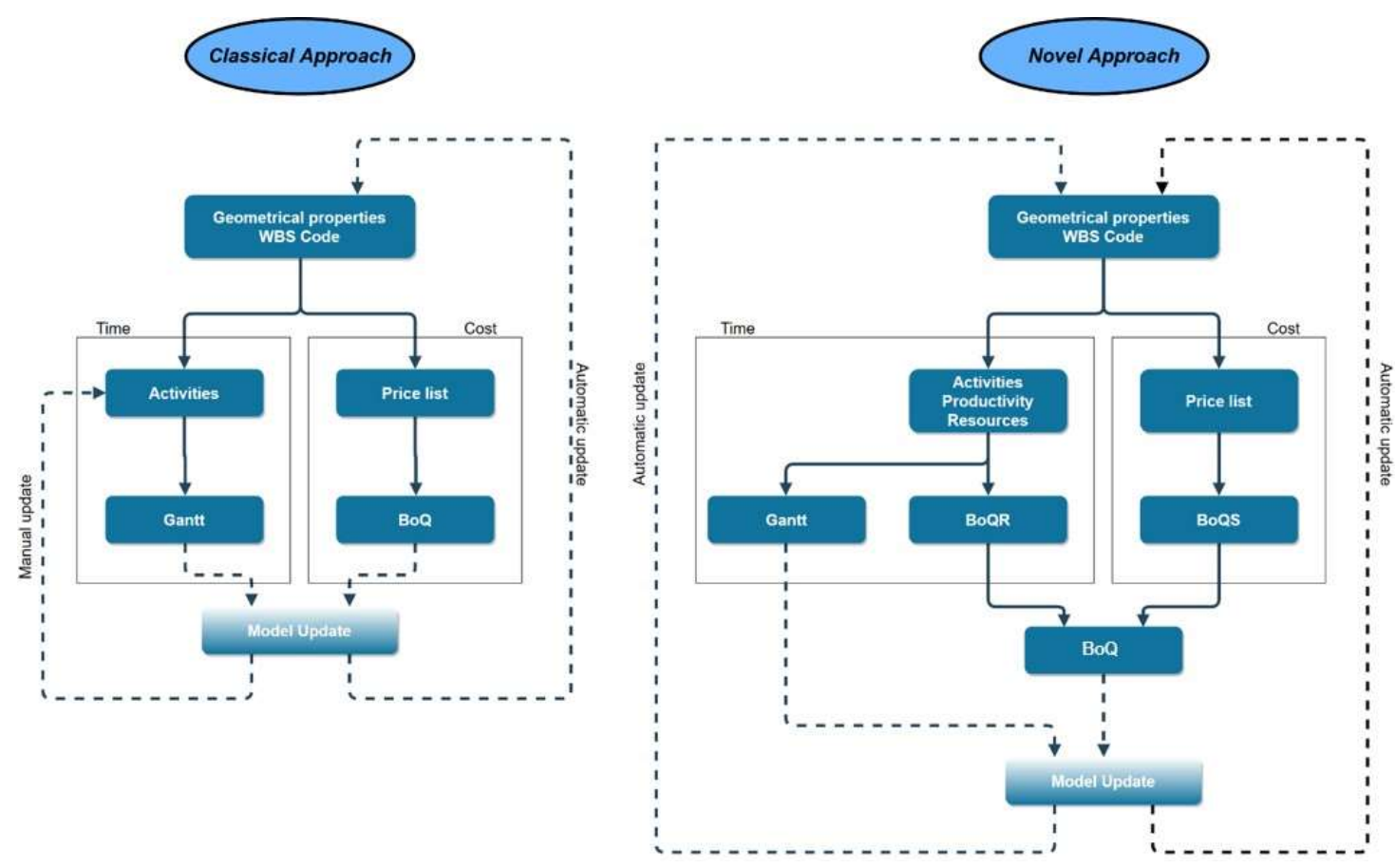

Figure 3: Comparison between flow-charts of classical (left) and proposed novel approach (right).

\section{Conclusions and future perspectives}

In this paper, a wide portion of the existing literature regarding the Building Information Modeling (BIM) approach is reported. In more detail, the fourth (4D) and fifth (5D) dimensions are here employed to explore new avenues for a better control of a project.

4D allows to link tasks in a project plan with 3D model components and compare different proposal plans by visualizing work sequences and duration of every task. This enables to: i) identify problems that may not be visible via traditional schedules; ii) run scenarios to assess feasibility of execution and find the best solution; iii) see the impact in 3D after updating the Gantt chart; iv) analyse how the build process will appear at different project stages; v) identify e solve potential dynamic interferences between the different teams on site.

Use of 5D simulation allows to generate models which identify how modifications on materials, layouts, constructive methodology and other design elements can affect the layout of an infrastructure as well as related costs. This is helpful to identify resource utilization over time.

Construction costs are modified automatically in real-time with the alterations made in the model. "What-if's" turns to be a productive and catching solution for the project team members in order to identify the best design layout.

A novel approach is proposed with an integration of time and costs in a unique twoway model within the context of a BIM process of a transport infrastructure. This is aimed at orienting the design process to the project optimization. The proposed model allows to dynamically update bill of quantities $(\mathrm{BoQ})$ and the time schedule as the model changes. In addition, once the various parameters and constraints have been defined, it provides further options for the designer to analyse more rapidly a higher amount of design alternatives. 


\section{References}

ACCA software (C) Primus CAD https://www.acca.it/software-cad-computo-metrico.

ACCA software (C) Primus IFC https://www.acca.it/software-ifc-bim-computo-metrico.

Autodesk (C) Civil 3D https://www.autodesk.com/developer-network/platformtechnologies/autocad-civil-3d.

Autodesk (C) Navisworks https://www.autodesk.it/products/navisworks/overview

Bentley System (C) OpenRoads https://www.bentley.com/it/products/brands/openroads.

Bentley System (C) SYNCHRO https://www.bentley.com/en/products/brands/synchro

Eastman, C., Teicholz, P., Sacks, R., Liston, K., 2011. BIM Handbook: a Guide to Building Information Modeling for Owners, Managers, Designers, Engineers and Contractors, BIM Handbook. John Wiley \& Sons.

EU, 2014. Directive 2014/24/EU of the European Parliament and of the Council of 26 February 2014 on public procurement and repealing Directive 2004/18/EC.

ISO, 2016. UNI EN ISO 16739, Industry Foundation Classes (IFC) for data sharing in the construction and facility management industries, Available online: https://www.iso.org/standard/51622.html.

Kalloc Studios (C) Fuzor https://www.kalloctech.com/

Lee, A., Wu, S., Marshall-Ponting, A., Aouad, G., Cooper, R., Tah, J., Abbott, C., Barrett, P. (2005) "nD modelling road map: A vision for $\mathrm{nD}$ Enabled construction", University of Salford, Manchester.

Lopez, R., Chong, H.-Y., Wang, X., Graham, J. (2016) "Technical review: analysis and appraisal of four-dimensional building information modeling Usability in construction and engineering projects", Journal of Construction Engineering and Management (ASCE), 142 (5), 1-6.

Lu, Q., Won, J., Cheng, J. C. P. (2016) "A financial decision making framework for construction projects based on 5D building information modeling (BIM)", International Journal of Project Management, 34(1), 3-21.

Matejka, P., Vitasek, S. (2018) "Comparison of different cost estimation methods with use of building information modelling (BIM)", Paper presented at the Engineering for Rural Development, 17, 843-849. doi:10.22616/ERDev2018.17.N154

Microsoft (C) MS Project https://products.office.com/it-it/project/project-and-portfoliomanagement-software.

MIT, (2017) "Ministero delle Infrastrutture e dei Trasporti, DM 560/2017: Modalità e i tempi di progressiva introduzione dei metodi e degli strumenti elettronici di modellazione per l'edilizia e le infrastrutture".

Mitchell, D. (2012) "5D BIM: Creating cost certainty and better buildings", Paper presented at the 2012 RICS Cobra Conference, Las Vegas, Nevada, United States of America.

OracleCPrimaveraP6, https://www.oracle.com/it/applications/primavera/products/project-portfoliomanagement.

Sacks, R., Gurevich, U. (2016), "A review of building information modeling protocols, guides and standars for large construction clients", Journal of Information Technology in Construction, 21, 479-503. 
SierraSoft (C) Roads http://www.sierrasoft.com/it/products/roads/roads.asp.

Team System (C) STR Vision CPM https://www.str.it/software/str-vision-cpm.

Wang, W.C., Weng, S.W., Wang, S.H., Chen, C.Y. (2014) "Integrating building information models with construction process simulations for project scheduling support", Automation in Construction, 37 Elsevier B.V., 68-80.

Zhang, J.P., Hu, Z.Z. (2011) "BIM- and 4D-based integrated solution of analysis and management for conflicts and structural safety problems during construction: 1. Principles and methodologies", Automation in Construction, 20 (2), 167-180.

\section{Acknowledgment}

The authors would like to thank Mr. Antonio Napolitano for the past and current assistance provided for the improvement of the proposed methodology. 